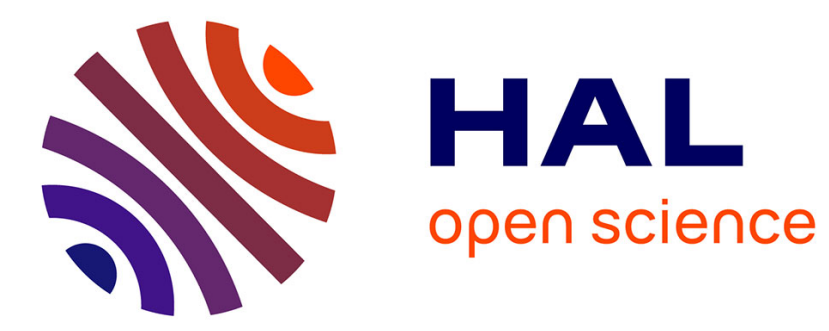

\title{
Inconscient et traduction, de la psychanalyse à la traductologie
}

Thamy Ayouch

\section{To cite this version:}

Thamy Ayouch. Inconscient et traduction, de la psychanalyse à la traductologie. L'Information Psychiatrique, 2009, Volume 85 (2009/2), pp.169-179. halshs-01003011

\section{HAL Id: halshs-01003011 https://shs.hal.science/halshs-01003011}

Submitted on 10 Jun 2014

HAL is a multi-disciplinary open access archive for the deposit and dissemination of scientific research documents, whether they are published or not. The documents may come from teaching and research institutions in France or abroad, or from public or private research centers.
L'archive ouverte pluridisciplinaire HAL, est destinée au dépôt et à la diffusion de documents scientifiques de niveau recherche, publiés ou non, émanant des établissements d'enseignement et de recherche français ou étrangers, des laboratoires publics ou privés. 


\section{Inconscient et traduction, de la psychanalyse à la traductologie}

\section{Thamy Ayouch}

Psychologue, psychanalyste

Maître de conférence à l'Université Lille 3, UFR de Psychologie, Domaine du Pont de Bois, BP 60149

59653, Villeneuve d'Ascq

20, rue Saint Sauveur, 75002, Paris

thamy.ayouch@gmail.com 


\section{Résumé :}

Inconscient et traduction, de la psychanalyse à la traductologie

Le processus de traduction accompagne la clinique analytique et sa théorisation dès les premiers textes de Freud : il fait le lien entre restes mnésiques, représentations de choses et représentations de mots, est décrit comme l'inverse du refoulement, ou constitue l'essentiel du travail du rêve. Réciproquement, le concept de traduction sert souvent à désigner le travail d'interprétation permettant d'accéder, depuis des images du rêve, des symptômes, des lapsus ou des actes manqués, à des pensées inconscientes. La question centrale de la traductibilité que la psychanalyse pose alors, dans un renvoi entre linguistique, herméneutique et métapsychologie, servira d'outil pour rechercher la nature de ce processus d' «acheminement à travers»(tra-ducere). Par le truchement de notions de traductologie, du concept merleau-pontyen d'expression, et de l'analyse de traductions de la première strophe du poème «The Raven » d'Edgar Allan Poe, nous interrogerons la nature linguistique de ce processus, et tenterons d'envisager une traduction sans langue originelle.

Mots-clés :

Traduction, inconscient, pluralité linguistique, expression, chiffrage, interprétation.

Summary :

Unconscious and Translation, from Psychoanalysis to Traductology

The process of translation appears in psychoanalytical clinical practice and theory already in Freud's early texts. Translation links mnemic residues, and things presentations to words presentations ; it is described as the opposite of repression, and underlays the major part of the dream-work. Yet reciprocally, this concept also refers to the interpretation-work that leads from dream images, 
symptoms, slips or parapraxes to unconscious thoughts. This central issue of traduceability, accross linguistics, hermeneutics and metapsychology, raises the question of the nature of this crossing process (tra-ducere). Through several notions of traductology, together with the merleau-pontyan concept of expression and the analysis of various translations of the first stanza of Edgar Allan Poe's « Raven », we will examine whether this process is linguistic and will try to conceive of some translation with no original language.

Key-words :

Translation, unconscious, linguistic multiplicity, expression, enciphering, interpretation.

Inconsciente y traducción : del psicoanálisis a la traductología

Palabras clave :

Traducción, inconsciente, pluralidad linguística, expresión, cifrar, interpretación 


\section{Inconscient et traduction, de la psychanalyse à la traductologie}

La notion de traduction semble revêtir une importance primordiale dans l'œuvre freudienne : elle accompagne la pratique clinique de Freud et son élaboration théorique dès les premiers textes. Deux vocables - Übertragung, Übersetzung - renvoient à de multiples sens de la traduction, qui s'articulent essentiellement dans deux directions, sans toutefois que l'emploi des termes allemands ne suive une systématicité stricte. En effet, hormis l'utilisation constante de Übertragung pour le phénomène de transfert que nous laisserons ici de côté, les deux termes Übertragung, Übersetzung se rapportent indifféremment aux occurrences multiples de la traduction dans et de l'inconscient. L'extension des termes en allemand est toutefois différente : déplacement à l'identique, telle la transposition musicale d'une tonalité à une autre, pour Übertragung qui suppose des règles fixes ; traversée d'une rive à l'autre pour l'Übersetzung, où l'Übersetzer est le passeur. Chacun à sa manière renvoie néanmoins, au latin traducere de Gellius, sur les modes objectif du transport pour Übertragung et subjectif du franchissement pour Übersetzung.

Deux processus globaux réciproques sont ainsi décrits de manière récurrente comme traductions : l'un a pour source l'inconscient, et pour résultats les différents effets de l'inconscient : rêves, lapsus, actes manqués, symptômes . L'autre, à l'inverse, a pour origine ces effets de l'inconscient, et pour cibles les pensées inconscientes dont ils résultent : il s'agit ici de l'interprétation, remontant du manifeste au latent. D'une traduction à l'autre, un retour à l'inconscient doublement original - originaire et excentrique. Dans l'espace de la séance analytique, entre ces deux traductions se tiennent l'analysé et l'analyste, que l'autre sens principal de Übertragung, transfert, vient relier. La pratique analytique pose alors la question centrale d'une traductibilité transversale aux niveaux topique, économique et herméneutique : qu'achemine-t-on (tra-ducere) ici, le fait- on nécessairement d'une langue vers une autre, est-ce en dernière instance un processus linguistique?

Penchons-nous plus précisément sur chacune de ses « traductions », pour vérifier la pertinence, dans la désignation de ces processus, de ce vocable essentiellement linguistique. 


\section{La traduction depuis l'inconscient}

\section{Les écritures mnésiques}

Dans les lettres à Fliess du 20 mai, et du 4 juin et du 6 décembre 1896, la traduction de traces mnésiques en images verbales désigne l'inscription d'une scène ou d'un événement dans le système mnésique de la conscience ${ }^{1}$. Reprenant les hypothèses de L'Esquisse d'une psychologie scientifique (1895) dans la lettre 52 ( 6 décembre 1896) Freud conçoit l'appareil psychique comme succession de systèmes entre lesquels s'effectue une traduction des matériaux psychiques [7]. Les névroses s'expliquent alors par «le fait que la traduction de certains matériaux ne s'est pas réalisée $»^{2}$, et ce défaut de traduction correspond au refoulement.

\section{$\underline{\text { Investissement économique et représentation de mot }}$}

La modélisation du rêve, dans la Traumdeutung et dans les textes ultérieurs, met au centre du travail du rêve la traduction «progrédiente », d'un système de l'appareil psychique à un autre système plus proche de la conscience. «Lorsque, écrit Freud, nous disons qu'une pensée inconsciente s'efforce de se faire traduire en préconscient pour pénétrer de force ensuite dans la conscience $»^{3}$, il s'agit d'un investissement ou d'un retrait de l'énergie à une organisation. Cette traduction n'est toutefois pas transmission, transport qui supprimerait du système original la pensée traduite, du fait de l'inscription en plusieurs endroits du système psychique ${ }^{4}$.

Dans le texte de 1915 consacré à l'inconscient, Freud souligne que le surinvestissement d'une représentation de chose, par sa liaison à des représentations de mots, dans le système Préconscient, introduit une organisation psychique plus élevée, rendant alors possible le remplacement du processus

\footnotetext{
${ }^{1}$ Selon une conception de l'appareil psychique que Freud redétaillera en 1925 dans la « Note sur le bloc-notes magique $»$.

${ }^{2}[8], \mathrm{p} 155$. Nous soulignons.

${ }^{3}[9], \mathrm{p} 518$. Nous soulignons.

${ }^{4}$ «Ce qu'il y a d'essentiellement neuf dans ma théorie, c'est l'idée que la mémoire est présente non pas une seule mais plusieurs fois, et qu'elle se compose de plusieurs sortes de 'signes' » rappelait Freud dans la lettre 52, in [8]., p 154.
} 
primaire par un processus secondaire. Dans les névroses de transfert, au contraire, « c'est la traduction en mots $»^{5}$ qui est refusée à la représentation de chose.

\section{$\underline{\text { La traduction dans le rêve }}$}

Au sein du rêve également a lieu une autre forme de traduction, celle de pensées inconscientes en images de rêve constituant l'essentiel du travail du rêve. Restes diurnes, souvenirs lointains, remaniements de scènes infantiles ou pensées inconscientes sont mêlés et déformés dans le rêve, qui emprunte, pour ce chiffrage, les processus de condensation, déplacement, et dramatisation.

«Les pensées du rêve et le contenu du rêve nous apparaissent, écrit Freud, comme deux exposés des mêmes faits en deux langues différentes: ou mieux, le contenu du rêve nous apparaît comme une transcription (Übertragung) des pensées du rêve, dans un autre mode d'expression, dont nous ne pourrons connaître les signes et les règles que quand nous aurons comparé la traduction (Übersetzung ) et l'original ${ }^{6 .}$

La métaphore linguistique est filée : Freud évoque deux langues différentes, l'enregistrement comme écriture, et le passage d'un texte à sa traduction. Le modèle heuristique prédéterminant dans cette conception est celui du déchiffrage, par Champollion, de la pierre de Rachid (Rosette). La comparaison des trois versions d'un même texte, la prière au pharaon Ptolémée $\mathrm{V}$, en écritures hiéroglyphique, démotique et grecque, permet à Champollion de percer le code de l'écriture hiéroglyphique. Double direction ici de la traduction, reprise par la psychanalyse dans sa double utilisation de cette notion : le Grec est traduction de l'écriture hiéroglyphique de la même manière que le contenu du rêve est traduction de ses pensées, mais, dans un second temps, c'est la comparaison entre ces traductions qui permettra à l'analyste, comme à l'archéologue, de parvenir à une traductioninterprétation des hiéroglyphes.

\section{Traduction entre symptômes}

Un usage différent du terme de traduction apparaît dans l'Interprétation des rêves. Au chapitre V, Freud évoque l'exemple d'une jeune fille, dont l'inimitié inconsciente à l'endroit de sa mère prend plusieurs formes : une aversion toute particulière, des rêves dont le sujet plus ou moins voilé est la

\footnotetext{
${ }_{6}^{5}[18], \mathrm{p} 117$. Nous soulignons.

${ }^{6}[9]$, p 241. Nous soulignons.
} 
mort de sa mère, et, ultérieurement, une phobie hystérique fondée sur l'idée qu'il puisse arriver quelque chose à sa mère. Ce sont ici, « comme traduits en plusieurs langages, simultanément, divers modes de réaction de l'appareil psychique à la même représentation émouvante ${ }^{7}$.

Dans Un souvenir d'enfance de Léonard de Vinci, cette traduction entre divers avatars des formations de l'inconscient est à nouveau évoquée. Le vautour penché sur le berceau de Léonard et qui introduit sa queue dans la bouche de l'enfant correspond à un souvenir écran, traduction à la fois du souvenir inconscient de l'allaitement par la mère, de la «trace indélébile » du fantasme d'un phallus maternel dans la théorisation sexuelle infantile, et d'un fantasme homosexuel de fellation passive.

\section{La traduction vers l'inconscient}

\section{$\underline{\text { Traduction du fantasme }}$}

Envisageons maintenant, à partir du Léonard, l'usage exactement réciproque de ce terme de traduction. Suggérant de traiter le souvenir de Léonard comme un rêve, Freud invite à traduire le fantasme qu'il contient, de son langage spécial en un langage généralement compris, et la traduction révèle une orientation érotique du souvenir : la queue «coda» du volatile vaut comme désignation substitutive du phallus ${ }^{8}$.

\section{La traduction des rêves : Traumdeutung, Traumübersetzung}

Le concept d'interprétation de la Traumdeutung s'érige précisément contre la traduction populaire des rêves, fondée sur une analogie symbolique et développée dans les mantiques. Si l'on excepte le sens des symboles sexuels - et le quatrième chapitre met toutefois en garde contre la réduction $\mathrm{du}$ « travail

\footnotetext{
${ }^{7}$ ibid. p 226

${ }^{8}$ Remarquons ici que toute l'interprétation du vautour dans Un souvenir d'enfance de Léonard de Vinci [18] et l'érudit développement sur la divinité égyptienne Mut, vautour femelle dotée d'un phallus, comme transposition de la théorie sexuelle infantile du phallus maternel, repose sur une erreur originelle de traduction interlinguistique : le terme nibio qu'emploie Léonard ne signifie pas vautour (Geier), comme le soutient Freud, mais milan. Voir à ce sujet le texte de Alan Bass «On the History of a Mistranslation and the Psychoanalitic Movement », in Difference in Translation, [24]
} 
de traduction du rêve à une traduction de symboles ${ }^{9}$ - Freud refuse toute transcription symbolique selon une «grammatologie » définie par avance, ou un langage symbolique pré-établi.

Le travail d'interprétation-traduction qu'il propose fait le cheminement inverse du travail du rêve, procède par associations libres survenant lors de la remémoration du rêve et fonctionne sur le modèle de l'archéologie. Dans le rêve, le contenu est donné «sous forme de hiéroglyphes dont les signes doivent être successivement traduits dans la langue des pensées du rêve ${ }^{10}$. Ainsi sont proposées des traductions des rêves de l'homme aux rats $^{11}$ ou de l'homme aux loups ${ }^{12}$.

L'interprétation d'un rêve articule donc trois types de traduction : la traduction du rêve en mots, la traduction symbolique d'éléments constants - les symboles sexuels - et la traduction-interprétation ${ }^{13}$.

\section{$\underline{\text { La traduction comme technique analytique }}$}

Cette traduction-interprétation est à la base de la technique psychanalytique. Elle contribue à faire entendre au psychanalyste l'étiologie des symptômes en révélant le matériel inconscient à leur fondement, mais prend aussi part à la cure, dans le cadre du transfert :

« La traduction de cet inconscient en conscient dans le psychisme du patient doit avoir pour résultat de ramener ce dernier à la normale et de supprimer la contrainte à laquelle est soumise sa vie psychique $»^{14}$.

Freud corrige par la suite cette idée que la simple révélation aux patients des pensées inconscientes sous-jacentes à leurs symptômes suffirait à les lever. La traduction des symptômes en pensées inconscientes oriente du moins le travail de la cure, et apparaît comme traduction conjointe du psychanalyste et de l'analysant.

\footnotetext{
${ }^{9}[9], \mathrm{p} 309$. Nous soulignons.

${ }^{10}[9], \mathrm{p} 241$

${ }^{11}$ Un rêve de l'homme aux rats dont le texte manifeste est « il voit ma fille devant lui, mais elle a deux morceaux de crotte à la place des yeux » reçoit comme «traduction »: «il épouse ma fille non pour ses beaux yeux mais pour son argent», in « Remarque sur un cas de névrose obsessionnelle. L'homme aux rats », in [14], p 229

${ }^{12}$ Freud propose de « traduire » la plainte d'être né coiffé de l'homme au loup en fantasme de fuite dans le corps maternel, in ibid. p 402

${ }_{13}^{2}$ [22], p 82.

${ }^{14}$ [11], p 20
} 
Afin de définir plus précisément la nature de ces processus de traduction, et d'analyser leur similitude avec la traduction linguistique, abordons maintenant quelques notions de traductologie.

\section{Traduction, langue et traductologie}

Le texte de Jakobson On Translation distingue trois formes de traduction: intralinguale «rewording» - , interlinguale, et intersémiotique - «transmutation». Dans les deux emplois complémentaires du vocable de traduction, renvoyant au mouvement progrédient de l'inconscient vers le conscient ou à l'interprétation de l'analyste et de l'analysant, est-il question d'un processus linguistique?

Observons d'abord que la traduction interlinguistique est explicitement évoquée par Freud, comme composante de certains processus inconscients ${ }^{15}$. En outre, Freud soutient que « les interprétations de la psychanalyse sont tout d'abord la traduction d'une modalité d'expression étrangère à nous en la manière d'expression familière de notre pensée ${ }^{16}$. Il s'agirait donc ici d'une traduction intersémiotique, entre le langage de l'activité psychique et celui de notre expression familière. La lecture lacanienne vient assimiler ce non-linguistique à une langue : la conception de l' "inconscient structuré comme un langage » prête au rêve la structure d'une phrase, et à l'organisation de ses éléments les lois de la rhétorique. Mais le modèle linguistique est ici étendu à l'ensemble de l'inconscient, et le primat du Symbolique, présent chez le premier Lacan « anté-topologique », fait d'emblée de ces traductions psychiques des phénomènes linguistiques. La question de leur nature semble donc ici tranchée d'avance, et c'est délibérément que nous laisserons de côté le modèle lacanien « anté-topologique », pour interroger la dimension linguistique de la traduction psychique.

\footnotetext{
${ }^{15}$ Dans la Psychopathologie de la vie quotidienne, l'oubli du nom Signorelli convoque, dans son mécanisme inconscient, la «traduction de Signor en Herr » ( [15], p 11). De même, Freud souligne, dans le cas d'un jeune Allemand, l'oubli de la traduction anglaise du mot « or » - pourtant identique en allemand - afin de pouvoir toucher la bague, et partant, la main d'une jeune fille anglaise. Dans Le délire et les rêves dans la Gradiva de W. Jensen, [16] c'est la traduction du nom de la jeune fille -Bertrang - et celle de son prénom - Zoé - qui permet à Norbert Hanold de l'assimiler inconsciemment à la Gradiva revidiva.

${ }^{16}[19], \mathrm{p} 198$.
} 
Pour examiner la dimension linguistique des traductions en psychanalyse, plutôt que de voir s'il s'agit de langue, tentons d'analyser si certains phénomènes que la traductologie associe à la traduction sont ici opératoires.

\section{Traductibilité relative et indétermination}

Que ce soit comme traduction-chiffrage (nous appellerons ainsi les diverses sortes de traduction depuis l'inconscient) ou traduction-interprétation (correspondant à la traduction vers l'inconscient), l'usage de la traduction par Freud semble radicaliser la théorie généralisée de la traductibilité relative de Quine : il n'y a pas de stricte correspondance entre l'original et sa traduction. C'est certes depuis une position épistémique bien différente que procède chacun de ces auteurs. Quine donne le primat à une conception béhavioriste de l'apprentissage de la langue, tributaire, selon lui, de la seule observation du comportement des autres. Hormis le comportement langagier publiquement accessible des locuteurs d'une langue, celle-ci ne comporte aucune signification fixée. Plus encore, la thèse de l'indétermination de la signification montre que l'observation du comportement langagier à elle seule ne peut définir de manière indubitable la signification d'une expression, et en proposer une traduction exacte dans une autre langue. Les "phrases observationnelles », voie d'entrée dans une langue étrangère qu'on s'essaie à traduire, sont sujettes à une indétermination quant-à leur sens, de même que les termes qui les composent sont indéterminés ${ }^{17}$. Jusqu'aux locuteurs natifs de la langue ne peuvent avoir raison de cette indétermination, qui commence dès l'apprentissage de la langue maternelle.

De même, dans notre conception psychanalytique des traductions chiffrage et interprétation, le textecible ne correspond jamais exactement au texte-source. Un même matériau inconscient peut revêtir diverses formes conscientes (la pluralité des mécanismes de défense est là pour en attester); une même formation de l'inconscient peut recevoir plusieurs interprétations. La différence qu'il convient toutefois de relever entre la perspective de Quine et celle de Freud tient à l'implication du « traducteur » dans le processus de traduction : pour l'analyste interprète, la traduction-interprétation

\footnotetext{
${ }^{17}$ Le fameux exemple du lapin développé dans Les Mots et les choses est parlant : si un locuteur prononce le mot « gavagai » à chaque fois qu'un interprète ne connaissant pas sa langue voit un lapin, l'interprète ne peut toutefois savoir avec certitude s'il s'agit ici du lapin, de l'être-lapin, du « segment continu de la fusion de tous les lapins », de l'étape de la vie d'un lapin, etc.
} 
qu'il formule ou à laquelle il amène l'analysant le convoque tout personnellement. Si le «texte source » reste impénétrable complètement, s'il est toujours susceptible de plusieurs traductions, c'est la perspective même de la cure, situation où l'analyste et l'analysant ébauchent conjointement une traduction-interprétation, qui détermine une traduction plutôt qu'une autre. Loin d'être un obstacle, cette «subjectivité » du traducteur est mise en exergue par Freud : l'interprétation convoque autant l'inconscient de l'analyste que celui de l'analysant.

Les traduction du chiffrage ou de l'interprétation dans la cure ressemblent ainsi aux échanges entre deux langues: il n'y a pas d'équivalence sans reste. Le français fou/fol qui traduit habituellement l'arabe مجنون écarte les associations dont bruisse ce terme, qui, dans sa racine verbale جن renvoie à l'occultation, au recouvrement et à l'obscurité, dans son radical جن désigne l'esprit, génie, entre-deux camouflé de l'homme et de l'âme et résonne alors comme possession par une puissance occulte. Sont laissés de côté le renvoi au paradis, جنة, Eden d'arbres cachant le sol de leur ombre, la référence à la sépulture, جنن, qui résonne de son antonyme fœtus, et l'allusion à l'esprit qui disparaît جن , ou à l'épanouissement d'un arbre qui fait apparaître ses bourgeons timides جن [les vocalisations sont à chaque fois différentes]. En outre, le vocable fol par son renvoi au latin follis, ballon, outre gonflée, remplace la dimension tellurique de جن par celle, aérienne, d’un esprit sans consistance et traversé de vent. Comme dans le passage d'une langue à l'autre, dans la transition du rêve à sa traduction en pensées inconscientes, il y a un reste irréductible, l' «ombilic du rêve », un hors-sens que toute interprétation ne saura faire reculer. Cela car, dans l'autre sens, la traduction-chiffrage des pensées inconscientes en images de rêve n'est pas totale et systématique. Mais ce reste irréductible dépend autant de l'inconscient de l'analyste que des points aveugles de l'inconscient de l'analyste.

Soulignons, en outre, la proximité des processus de l'inconscient et des déformations de la lettre du texte original, que Berman rappelle dans le texte «La traduction et la lettre ou l'auberge du lointain $»^{18}$. La traduction de et vers l'inconscient emploierait les mêmes « ratages » propres à la traduction interlinguistique.

\footnotetext{
${ }^{18}$ Ainsi donc, la rationalisation, le passage de la polysémie à la monosémie, l'ennoblissement, l'appauvrissement quantitatif, l'homogénéisation, la destruction des réseaux sous-jacents, ou la destruction des locutions, sont
} 


\section{Traduction et Unheimlich}

L'analogie entre traduction linguistique et traduction psychique peut être poursuive. Il arrive parfois qu'un traducteur inter-linguistique se retrouve projeté au plus près de l'étymon, au plus loin du sens, et, dans une sidération devant le signifiant, s'arrête, frappé par l'immotivation, dans la langue d'origine et celle d'accueil. De même, devant l'unheimlich des processus inconscients, l'analysé peut rester sans mots pour « traduire » son rêve, il peut recevoir de l'analyste une traduction-interprétation dans l'ambivalence qui caractérise la Verneinung: acceptation intellectuelle du refoulé, mais refus affectif, par un maintien du refoulement ${ }^{19}$.

Ignorant dans quel espace linguistique il se situe, le traducteur vient parfois à connaître un moment de suspens interlinguistique ; il peut, dans la conception métaphysique de Walter Benjamin, transiter par un état hors langue, mythe heuristique d'une langue sacrée qui anticipe le «règne de la langue pure $»^{20}$. De même, l'analysé peut se retrouver dans les limbes intersémiotiques séparant l'interprétation d'une part, du langage du rêve, de la névrose, ou de l'acte manqué d'autre part. N'inversons toutefois pas ici les résultats de l'analyse. Si le traducteur connaît ce saisissement où des éléments d'une langue d'origine ou d'accueil s'éclairent violemment et ne semblent plus naturels, c'est parce que le phénomène même de la traduction interlinguistique convoque des processus inconscients. Dans l'espace de l'interlangue a lieu une expérience unique, comme le commente Jacques Hassoun dans L'Exil de la langue, l' «advenue du sujet tel qu'il se situe dans son adresse à l'Autre $»^{21}$. Ce n'est pas ici la traduction-chiffrage ou la traduction-interprétation de l'inconscient qui présentent des caractères propres à la traduction interlinguistique, mais plutôt celle-ci qui participe de l'inconscient.

autant de procédés du passage d'une langue à l'autre, qui valent également lors de la conversion des processus primaires en processus secondaires, dans la traduction-chiffrage. Inversement, dans le cas de la traductioninterprétation, la traduction d'un matériel manifeste en pensées latentes s'accompagne d'un allongement ( on défait les condensations du rêve), d'un appauvrissement qualitatif (suppression de l'iconicité du rêve), d'une destruction des rythmes ou des locutions, autant de tendances déformantes propres à la traduction linguistique ( in [4])

${ }^{19}$ Cf. Le texte de Freud « La Négation ».

20 [2].

21 [27], p 232 


\section{Traduction : l'original augmenté}

Poursuivons toutefois notre comparaison. Selon Walter Benjamin, ce n'est pas tant la traduction qui enrichit la langue d'accueil, que ce passage par la langue d'accueil qui révèle de nouvelles dimensions du texte source. De même, comme le souligne Freud dans «Remémoration, répétition, et perlaboration $»^{22}$, la communication de l'interprétation-traduction, conjuguée au travail sur les résistances modifient la dynamique des systèmes psychiques et le jeu de leurs forces. La traductioninterprétation vient combler les lacunes de la mémoire, vaincre les résistances du refoulement: s'ensuit une transformation de l'inconscient, remplaçant la répétition par un ressouvenir, et la résistance par une perlaboration.

Si, pour Benjamin, la tâche du traducteur consiste à délivrer dans sa langue la langue pure exilée, capturée, dans l'œuvre étrangère, à faire advenir la langue originaire cachée, messianique langue postbabélique, la tâche de l'interprète de l'inconscient vise, elle aussi, la libération de cette langue originaire des pensées latentes et du fantasme.

Le «texte» initial de l'inconscient ne cesse de se remodeler au gré de ses successives traductions (interprétations), dans une conception herderienne de la traduction comme transplantation d'un auteur sur le sol d'une nouvelle langue. Le résultat de cette entreprise est double : la traduction change l'œuvre, lue alors à d'autres ères et en d'autres contrées, mais elle modifie également la langue d'accueil, en l'infusant du style de l'auteur traduit. De même, la traduction-chiffrage d'une pensée inconsciente, comme dans le cas de l'adversité envers la mère évoquée précédemment ${ }^{23}$, présente, dans la succession des symptômes différents - aversion motrice, rêves de mort, phobie hystérique des « lecture» différentes d'un même «texte» qui en renouvellent les ressources. Cependant, à la différence des images du rêve de mort ici, qui sont une traduction-chiffrage de représentations inconscientes advenant à la conscience, les divers symptômes sont des traductions entre eux, mais strictement parlant, ne correspondent pas à la traduction de représentations inconscientes : ils en sont les déformations, autant d'échecs de traduction provoquant la névrose. Il y a donc ici éclatement de

\footnotetext{
${ }^{22}[13]$.

${ }^{23}[9], \mathrm{p} 226$
} 
ces divers textes d'arrivée : texte « réussi », lorsqu'il s'agit de la traduction de pensées inconscientes dans des images de rêve, texte "raté », dans le cas du symptôme névrotique et toutefois bien réel, comme en témoigne la souffrance qu'il provoque. En outre, il n'y a pas ici de langue d'arrivée, puisque dans le cas de la traduction « réussie », les images de rêve, les représentations de mots ou les enregistrements mnésiques ne viennent pas s'inscrire dans une langue unifiée quelconque, leur préexistant. C'est dans leur apparition même qu'ils créent la langue les exprimant.

\section{L'indissociabilité des « langues »}

Le résultat semble fondamental: dans les cas de la traduction-chiffrage comme de la traductioninterprétation, même à supposer l'existence d'une métaphorique langue source, force est d'admettre qu'il n'y a pas de langue cible, susceptible d'être influencée par le procédé même de la traduction. Il semblerait possible de parler dans la traduction-chiffrage de «langue source »- les processus primaires - et de «texte source»- les traces mnésiques, pensées inconscientes ou représentations de mots. Si le texte cible est l'inscription des traces mnésiques dans un système supérieur - les images de rêve ou les représentations de mots - il n'y a toutefois pas de langue cible pré-existant à ce texte. Les systèmes psychiques ne sont pas des langues mais des lieux d'inscription, il n'y a pas de langue des processus secondaires indépendamment de ces processus secondaires eux-mêmes. Ils sont cette langue, ou du moins cette langue n'est qu'eux : contrairement à l'espagnol par exemple, qui ne se réduit pas à la traduction par Julio Cortazar des Mémoires d'Hadrien, ni à toutes les traductions de textes écrits dans d'autres langues.

Symétriquement, la langue source de la traduction-interprétation est inexistante en elle-même, et se fragmente en autant de textes sources différents : les représentations de mots, les images de rêve, les traces mnésiques supérieures. En outre, la langue linguistique de l'interprétation n'est pas modifiée par l'interprétation - donc par la traduction, - mais au contraire par la non-interprétation, dans le symptôme linguistique qu'est le lapsus par exemple. Elle est langue exprimant l'interprétation, et non point langue de l'interprétation comme le français serait la langue de la traduction baudelairienne de Poe. Mais par ailleurs, il apparait clairement que ce que nous postulions comme langue source de la traduction-chiffrage - celle des processus primaires - reste inaccessible, et que la dénomination de 
« langue » n'est que convenance ici. La langue des processus inconscients renvoie au fantasme d'une première langue originaire, anté-linguistique, maternelle, irrémédiablement perdue et qu'aucune traduction ne retrouvera.

A considérer les processus primaires comme langue source de la traduction-chiffrage, la langue cible les processus secondaires - se réduit à la multiplicité des textes cibles, elle disparaît comme langue cible en soi, ou ne tient comme telle qu'à être conçue comme langue cible pour une hypothétique langue source incarnée par les processus primaires. De même, dans l'autre sens, à prendre acte de l'éclatement de la langue-source de la traduction-interprétation, la langue-cible des processus primaires apparaît illusoire : cette asymptotique langue de l'inconscient n'aurait d'existence propre qu'en étant idéalement visée comme langue cible, pour l'interprète, d'une pluralité de manifestations, elles-mêmes unifiées sous une inexistante langue commune des processus secondaires. Qu'est-ce à dire ici, sinon que dans la traduction-chiffrage autant que dans la traduction-interprétation, il n'y a pas de langue source indépendante d'une langue cible, ni de langue cible concevable sans une langue source? En d'autres termes, il n'y a pas dualité ontologique et temporelle entre la langue source et la langue d'accueil : les processus secondaires traduisant les processus primaires n'existent pas hors d'eux, ils ne sont là que grâce à la traduction ; inversement, les pensées latentes auxquelles parvient l'interprétation ne préexistent pas au matériel manifeste qui les traduit, et n'adviennent à l'être que du fait de la traduction-interprétation de l'analysé et de l'analyste.

C'est là une critique fondamentale qu'adresse la phénoménologie au modèle de la traduction dans l'inconscient.

\section{Une traduction non linguistique? L'expression et ses limites}

\section{L'expression phénoménologique}

La critique phénoménologique de cette conception traductive de l'inconscient revient à Maurice Merleau-Ponty, dont la lecture de la psychanalyse reste ici influencée par celle de Politzer ${ }^{24}$. Pour

\footnotetext{
${ }^{24}$ Dans la Critique des fondements de la psychologie, [39], Politzer soutient qu'il n'y a pas d'antécédence du latent sur le manifeste: au contraire, les pensées latentes sont construites après coup par l'analyse et l'interprétation, et incorrectement postulées à l'origine des processus manifestes.
} 
Merleau-Ponty, rêver n'est pas traduire un contenu latent dans le langage clair du contenu manifeste, c'est le vivre à travers le contenu manifeste ${ }^{25}$. Le «symbolisme primordial ${ }^{26}$ du rêve est ce qui permet, à travers le contenu manifeste, de viser le contenu latent, non point par traduction de l'un dans l'autre, mais en le vivant, dans une présence qui est absence et constitue le mode d'être de l'inconscient. Le passage de l'un à l'autre de ces plans est réalisé par la catégorie de l'expression où se confondent signe et sens. Le contenu manifeste ne vient pas traduire dans le rêve un contenu latent qui demeure caché, ni la généralité de notre corps une sexualité cachée, mais en sont les expressions.

L'inconscient qui détermine la dialectique entre sexualité et existence, passé et présent, se lit donc dans ce rapport d'expression. En ce sens, il n'est pas profondeur cachée, mais reste conçu dans la continuité d'une conscience qui n'est plus alors positionnelle, thématisante, mais «rêveuse ». Pour cette conscience, le sens advient non point dans l'évidence d'une donation originaire, mais indirectement, et de par la généralité du corps.

Cette vision se trouve radicalisée par exemple dans la note de travail du 2 mai 1959 du Visible et l'invisible, ou Merleau-Ponty supprime toute dimension de traduction inter-linguistique dans les phénomènes de l'inconscient, en les comparant paradoxalement à l'expérience de confrontation à une langue étrangère. Il présente deux phrases entendues à Manchester ${ }^{27}$, qui ne sont comprises que quelques secondes après leur prononciation. Leur sens advient d'un coup, par retour ou « mouvement rétrograde $»^{28}$ : il y a une première perception, conçue comme «germination de ce qui va avoir été compris » dans une deuxième perception. Cette première perception est comparée à l'inconscient, ouverture d'un champ de Gestaltungen, pivot, existential, qui est et n'est pas perçu, et qu'un retour vient confirmer. En ce sens, l'inconscient comme matériel latent est ici perception entre les objets, et le manifeste n'advient pas comme une traduction séparée, mais comme la perception des objets, solidaire de cette perception-imperception entre les objets. L'interprétation de l'inconscient reprend cette compréhension d'une langue étrangère : un sens non reconnu mais, en fait, déjà perçu vient

\footnotetext{
${ }^{25}[40], \mathrm{p} 70$.

${ }^{26}$ ibid.

${ }^{27}$ Celle d'un chauffeur de taxi, «I'm going to ask the police where Brixton Avenue is », et celle d'une vendeuse, «Shall I wrap them together?».

${ }^{28}$ [39], p 242-243
} 
modeler et diriger ma reconnaissance ultérieure des mots de la langue, dans une solidarité entre cette perception-imperception latente et la perception manifeste. Les phonèmes d'abord non séparés le sont immédiatement après, non pas par une traduction ici des phrases anglaises en français, mais dans l'expressivité qu'elles détiennent après-coup.

\section{$\underline{\text { La critique des psychanalystes }}$}

La continuité entre manifeste et latent, à travers la catégorie d'expression, implique donc ici une problématique continuité entre inconscient et conscience. C'est ce qui fera l'objet des critiques conjointes de psychanalystes tels Jean-Bertrand. Pontalis et André Green ${ }^{29}$.

Sous la catégorie trop générale du sens, affirme J. B. Pontalis, Merleau-Ponty amalgame relation expressive - où la signification est immanente à l'objet - et relation linguistique - reposant sur la différence entre signifiants - dans une confusion réelle, bien que délibérée, entre les notions de structure et de signification.

Il y aurait primat, chez Merleau-Ponty, du modèle perceptif, sur tout modèle linguistique. Le modèle freudien du rêve n'est toutefois pas, souligne J.B. Pontalis, un modèle de l'expression, mais de la traduction : le rêve est rébus, l'image n'y vaut pas comme visuel mais comme signe, et si elle offre un sens, ce sens cache le signe ${ }^{30}$.

Plus sévère est encore la critique de Lacan $^{31}$, qui dénonce lui aussi l'absence de dimension symbolique propre chez Merleau-Ponty. La phénoménologie « se condamne à la fois à déborder de son champ et à se rendre inaccessible une expérience qui lui est étrangère $»^{32}:$ la structure de la réalité n'y est pas

\footnotetext{
${ }^{29}$ Cf. les textes J.B. Pontalis, «La Position du problème de l'inconscient chez Merleau-Ponty » [40] et «Présence, entre les signes, absences » [39]; A.Green « Du comportement à la chair : itinéraire de MerleauPonty » [25].

D'autres psychanalystes adressent à Merleau-Ponty une critique similaire: Cf. les textes Jacques Lacan, "Maurice Merleau-Ponty », in Autres écrits, Seuil, Paris, 2001; Cornelius Castoriadis, "Merleau-Ponty et le poids de l'héritage ontologique », in Fait et à faire, Seuil, Paris, 1997, pp 157-194.

${ }^{30}$ J.-B.Pontalis, « Perdre de vue », in [40]

${ }^{31}$ Lacan évoque Merleau-Ponty à bien des reprises dans son Séminaire. Il lui accorde une attention plus précise dans l'hommage écrit à la mort du philosophe en 1961 [29], où il fait état de ses principales critiques à son endroit. Il les reprend dans le onzième livre du Séminaire [30].

${ }^{32}$ J. Lacan, [29].
} 
conçue comme articulation de signifiants. La phénoménologie se cantonnerait alors au seul plan de l'Imaginaire, présupposant dans le corps un lieu de l'unité.

Plus tard, dans le livre XI du Séminaire, Lacan rapporte au Visible et l'invisible l'intuition fondamentale de la structure même de la vision telle qu'elle articule l'objet a dans l'Imaginaire. Lors de la séance du 19 février 1964, il souligne que Merleau-Ponty «forçant les limites de la phénoménologie ${ }^{33}$, désigne la dépendance du visible «à l'égard de ce qui nous met sous l'œil du voyant»: la préexistence du regard. Encore une fois, si le philosophe semble appréhender indirectement la fonction du Symbolique, il reste cantonné à l'Imaginaire. La dimension qui manquerait à Merleau-Ponty pour articuler le Symbolique ne serait pas à trouver du côté du corps, de la conscience ou de la perception, elle relève de l'inconscient, selon Lacan.

Il n'y a donc pas, chez Merleau-Ponty, d'univers symbolique distinct, l'inconscient n'est pas une batterie de signifiants séparés, là où au contraire, conscient et inconscient sont deux systèmes distincts, topiquement et dynamiquement opposés, séparés par la censure ${ }^{34}$.

\section{Continuité, distinction et structure topologique}

Notre propos n'est pas ici de discuter cette critique - il conviendrait, à ce sujet, de voir que l'imaginaire prend un sens bien distinct en phénoménologie et dans la théorie lacanienne, mais aussi que la phénoménologie de la chair du dernier Merleau-Ponty permet de confirmer l'entrelacs entre perception et désir propre à la formation de la réalité. Nous croyons toutefois que la thèse merleauponytenne d'une indissociabilité de la conscience et de l'inconscient, et partant, de la source et de la cible dans la traduction psychique, trouverait une résonance symbolique dans la topologie lacanienne. En prenant la modélisation topologique du Symbolique plutôt que la précédente structuration de l'inconscient «comme un langage», nous ne tranchons pas de prime abord la question qui ici dirige notre recherche : celle d'une dimension linguistique de la traduction psychique. En ce sens, deux figures de la topologie, la bande de Möbius et de la bouteille de Klein, offrent une « esthétique transcendantale » de la traduction psychique : elles permettent notamment de résoudre les apories des

\footnotetext{
${ }^{33}$ J. Lacan, [30], p 84.

${ }^{34}$ Cf. [20].
} 
oppositions entre dedans et dehors, processus primaires et processus secondaires, représentations de mot et représentations de chose, «textes » source et cible. Figuré par la bande de Möbius, le psychisme apparait dans sa division entre conscience et inconscient - envers et endroit du ruban - qui ne s'en rejoignent toutefois pas moins, dans la solidarité entre langue-source et langue-cible, pour la traduction-chiffrage comme pour la traduction-interprétation. Par la bande de Möbius, les processus secondaires traduisant les processus primaires dans la traduction-chiffrage en sont indissociables, et, inversement, les pensées latentes que révèle la traduction-interprétation sont solidaires de leur verbalisation manifeste : l'envers et l'endroit sont à la fois opposés et se rejoignent. L'intérêt de cette figure topologique est de faire apparaître l'acte de langage comme coupure, garante de la schize entre conscience et inconscient. C'est par l'acte de langage que les représentations de choses, primaires, s'associent à des représentations de mots, secondaires, dans la traduction-chiffrage, et c'est dans l'acte même de verbalisation d'une traduction-interprétation qu'apparaît la dualité de la pensée latente et de sa traduction manifeste. Coupure du ciseau, l'acte de langage restaure ses deux bords au ruban de Möbius : il crée un texte source et un texte cible, différents, mais indissociables sans l'intervention du langage. En ce sens, si la traduction psychique se voit dotée d'une dimension linguistique, c'est dans la coupure signifiante de l'acte de langage propre à la traduction-chiffrage (association de représentations de mots à des représentations de choses) et à la traduction-interprétation (désignant un matériau latent sous le manifeste).

\section{Traductions en psychanalyse, psychanalyse des traductions}

Il reste donc légitime de parler de traduction pour le chiffrage et l'interprétation: la critique de Merleau-Ponty par les psychanalystes vient restaurer la séparation radicale des systèmes inconscient et conscient, et l'acte de langage entérine la dimension linguistique de ce processus psychique.

Pour conclure sur cette dimension linguistique, nous proposons d'analyser quelques traductions de la première strophe du poème «The Raven » d'Edgar Allan Poe, à travers diverses langues. Le choix 
d'une analyse de poème nous semble ici adéquat à la difficulté à laquelle nous sommes parvenus.

Nous distinguerons, comme le fait la psychanalyse, un contenu latent du poème et un contenu manifeste. Toutefois, concession faite ici à la phénoménologie et à la topologie, malgré la séparation de ces deux plans, il ne s'agit pas de deux niveaux ontologiquement distincts, et dont l'un précéderait l'autre. Dans la tentative de psychanalyse littéraire que nous effectuerons, l'inconscient est celui du poème - nulle question d'évoquer ici un quelconque inconscient de l'auteur. En ce sens, il n'y a pas d'inconscient sans poème : l'inconscient est contemporain ici de sa propre manifestation, et n'existe pas hors d'elle.

Examinons ici la première strophe du poème et quelques unes de ses traductions :

« Once upon a midnight dreary, while I pondered, weak and weary, Over many a quaint and curious volume of forgotten lore, While I nodded, nearly napping, suddenly there came a tapping, As of some one gently rapping, rapping at my chamber door. "'Tis some visitor," I muttered, "tapping at my chamber doorOnly this, and nothing more." »

Traduction française, Nicolas Abraham :

"L'heure d'une minuit sourde, lorsque l'âme lasse et lourde, J'eus fouillé dans maint bizarre et vieux volume aux morts trésors, Tandis que je songe, inerte, brusquement se fait un heurt, On eût dit : quelqu'un qui heurte, heurterait à l'huis dehors. «C'est quelque hôte qui - soufflé-je - heurte à peine à l'huis dehors, Oui, le reste est sans rapport »».

\section{Traduction portugaise, Fernando Pessoa :}

Numa meia-noite agreste, quando eu lia, lento e triste, Vagos, curiosos tomos de ciências ancestrais,

E já quase adormecia, ouvi o que parecia

O som de algúem que batia levemente a meus umbrais.

«Uma visita", eu me disse, "está batendo a meus umbrais.

É só isto, e nada mais."

Traduction italienne, Francesco Contaldi

Mentre, debole e stanco, verso la mezzanotte scorrea d'antico libro pagine strane e dotte sonnecchiando, ad un tratto come un picchio ascoltai, un lieve, un gentil picchio de la mia stanza all'uscio. - E' qualcuno che picchia de la mia stanza all'uscio, e non altro, - pensai.

Traduction espagnole, Juan Antonio Pérez Bonalde : Una fosca media noche, cuando en tristes reflexiones, Sobre más de un raro infolio de olvidados cronicones 
Inclinaba soñoliento la cabeza, de repente

A mi puerta oí llamar; como si alguien, suavemente,

Se pusiese con incierta mano tímida a tocar:

"¡Es - me dije - una visita que llamando está a mi puerta:

eso es todo y nada más!".

Traduction catalane, Miquel Forteza :

Una trista mitja nit, que vetllava entenebrit,

fullejant amb greu fadiga llibres vells i antics papers

i em dormia a poc a poc, vaig sentir a la porta un toc.

I sens moure'm del meu lloc: "Qualcú ve a cercar recés

-vaig pensar- en aquesta hora, qualcú ve a cercar recés."

Això sols i no res més.

Dans son texte « Pour une esthétique psychanalytique : le temps, le rythme et l'inconscient » ${ }^{35}(1962)$,

Nicolas Abraham présente une lecture psychanalytique de cette strophe dont nous reprenons ici les résultats. La discordance sémanto-rythmique, entre la répétition simple de trochées, monotones, véhiculant une tentative d'endormissement et l'incident nouveau, récurrent, de la visite et des coups vient montrer que la réalité qui fait irruption n'est pas un événement extérieur mais un vœu angoissant, présent sur un mode hallucinatoire. Si donc le niveau manifeste est ici perte d'un objet - la Lenore disparue - le niveau latent dévoile un fantasme nécrophile d'incorporation de l'objet perdu, fantasme satisfait, car l'objet est réintroduit à jamais dans le moi à l'instar du corbeau qui ne quittera jamais plus (« Nevermore ») la chambre.

Attachons-nous à retrouver cette tension entre niveaux manifeste et latent en faisant jouer entre elles les différentes traductions. Contentons-nous d'analyser trois points ici, qui confirment l'ambivalence perte/incorporation décelée par N. Abraham.

Dans la plupart des versions, le rythme binaire de répétition de trochées reste globalement le même que dans le texte original, et reflète la régularité par excellence. Seule exception ici, la version italienne alterne des trochées et des dactyles (Mentre,/ debole e /stanco, /verso la /mezza/ notte). Avec la catalane, elle présente une irrégularité majeure dans le dernier vers :

e non $\mathbf{a l} /$ tro, - pensai (anapeste anapeste)

Això /sols i no / res més (iambe, dactyle, spondée).

${ }^{35}[1]$. 
Dans ces deux traductions, le dernier vers vient briser complètement le rythme en l'inversant. A cette inversion rythmique fait pendant une opposition sémantique : non point au cœur d'une version, sols (seulement), et res més (rien de plus) étant deux pieds dont le sens reste le même, mais entre deux langues: le contenu sémantique de altro (autre) étant contredit par celui de sols et de res més. L'opposition de ces deux versions à l'ensemble des autres, et entre elles par ce dernier point, vient accentuer l'ambivalence qui règne entre niveaux manifeste et latent, comme si la perte incorporation venait résonner, par la mise en perspective des différentes traductions, en présence/absence, même et autre.

Un jeu de rimes exactement identiques se retrouve entre l'original, le français, le portugais, et le catalan :

$\begin{array}{ll}\text { a1 a2 } & \text { dreary/weary } \\ \text { x b1 } & \text { curious/lore } \\ \text { c1 c2 } & \text { napping/tapping } \\ \text { c3 b2 } & \text { rapping/door } \\ \text { x b3 } & \text { muttered/door } \\ \text { b4 } & \text { more }\end{array}$

A travers les rimes b, dans l'original, (lore/door/more), le savoir reste à la porte (door), de même qu'en portugais (ancestrais / umbrais / mais) cet héritage originaire et ancien (ancestrais) demeure au seuil (umbrais), alors qu'en français (trésors/dehors/rapport) il est mis dehors, et qu'en catalan (papers/recés/més), au contraire, il est reclus (recés), inclus à l'intérieur. C'est en faisant jouer les langues entre elles qu'apparaît au mieux cette dialectique d'inclusion/exclusion et seuil propre à l'ambivalence d'une perte et réincorporation.

Enfin, à un niveau sémantique, il y a opposition, au sein de l'original, entre dreary et weary, exprimant la monotonie et l'ennui d'une part, et tapping rapping qui marquent l'incident nouveau d'autre part. Cette opposition est au fondement de l'hallucination d'une présence, présence/absence qui renvoie à la perte/réincorporation. C'est en confrontant les avatars de ces deux lignes sémantiques dans les diverses traductions que se confirment cette perte/réincorporation comme présence/absence. Le minuit dreary a pour « équivalents » dans les traductions l'étouffement de « sourde », la grossièreté tactile du 
portugais agreste (rustique, rude), aucune épithète en italien, la répulsion visuelle de l'espagnol fosca (ténébreux, rébarbatif) et l'abattement du catalan trista, dans une convocation de différent sens (ouïe, toucher, vue) et de leur absence qui réaffirme le thème de l'hallucination sensitive. La ligne de tapping (donner des petits coups) associe aux connotations de violence du batia (battait) portugais et picchio (coup) italien (tous les deux pouvant renvoyer à des coups portés à des choses ou à des personnes) les sensations auditives de l'espagnol llamar (appeler, frapper à la porte) et auditivo-tactiles du catalan toc (coup sur la porte et bruit), conjuguant ici aussi le tactile à l'auditif. Observons en outre que picchio désigne également un oiseau en italien, ce qui vient augurer de l'intrusion du corbeau. La révélation de l'hallucination se confirme avec la ligne de rapping. Y correspondent « heurte» en français, picchio en italien, tocar (toucher) en castillan, et l'absence de signifiant en portugais et en catalan. A la place de ces deux béances, deux propositions qui n'existent pas dans l'original. Le mirage du portugais « $O$ que parecia» (ce qui semblait) et l'immobilisme du catalan «sens moure'm del meu lloc» (sans me mouvoir du lieu ou j'étais) viennent renforcer l'idée d'une hallucination sensitive et motrice. La sensation d'hallucination est donc entérinée par la mise en perspective de ces différentes versions.

Qu'est-ce à dire ici sinon que ces diverses traductions inter-linguistiques reprennent l'éclatement des traductions-chiffrage de l'inconscient : le français, le portugais, l'italien, l'espagnol ou le catalan sont autant d'avatars manifestes de l'inconscient. En retour, l'acte de langage par lequel nous relions entre elles ces traductions est semblable à l'interprétation en cure : il vient pointer les pensées inconscientes du poème en les rendant solidaire, dans leur verbalisation, de ce système entrecroisé de traductions, et introduit ainsi une dualité.

Il n'y a donc pas, dans la traduction de l'inconscient - traduction-chiffrage ou traduction-interprétation - de langue source indépendante d'une langue cible, ni de langue cible non tributaire d'une langue source. Pensées inconscientes et images du rêve, traces mnésique et leur inscription dans un système supérieur, représentations de chose et représentations de mot, latent et manifeste, sont en ce sens solidaires les uns des autres, plus étroitement que tout texte cible d'un texte source dans une traduction interlinguistique. S'il y a traduction dans et de l'inconscient, elle n'est donc pas linguistique au sens où les niveaux latent et manifeste correspondraient à deux langues indépendantes, mais au sens où elle 
advient par l'acte de langage qu'est le chiffrage ou l'interprétation. De cette façon, elle convoque plusieurs langues. De même que les différentes manifestations de l'inconscient sont traductions les unes des autres, et renvoient, seulement lorsqu'elles sont toutes mises en perspective, à un même fond, de même, le fond inconscient du poème de Poe n'apparaît qu'en mettant en perspective ses différentes versions linguistiques. La langue archaïque n'est donc pas une quelconque origine génétique, mais l'articulation, diacritique, de tous les textes qui y renvoient, des images de rêve, des lapsus, et des symptômes d'un même sujet, de même que la langue inconsciente, anté-babélique du texte de Poe, n'advient que de la dédifférentiation des diverses langues dans lesquelles il est traduit.

Tel le plurilingue drogman oriental, «tourdjouman», dont l'étymologie hébraïque [גרת [tirguem] souligne la dimension d'action, et l'étymologie arabe ترجم [tarjama] celle d'écriture biograpique, la traduction psychique est mise en acte du primaire devenu secondaire par le chiffrage, mais aussi une constante écriture de soi par l'élaboration que permet l'interprétation. Elle est ainsi traduction plurilinguistique, et cette pluralité la fait, disait Hölderlin, « tel un incendie, éclater entre la confusion des langues ». 


\section{Bibliographie}

1. ABRAHAM, N., "Pour une esthétique psychanalytique : le temps, le rythme et l'inconscient », in Rythmes de l'œuvre, de la traduction et de la psychanalyse, textes recueillis et présentés par N. T. Rand et M. Torok, Paris, Flammarion, 1985.

2. BENJAMIN, W., «La tâche du traducteur », in Euvres 1, trad. Maurice de Gandillac, Rainer Rochlitz et Pierre Rusch, Paris, Gallimard, 2000.

3. BERMAN, A., L'Epreuve de l'étranger, Paris, Gallimard, 1984.

4. BERMAN, A., « La traduction et la lettre ou l'auberge du lointain », in Les Tours de Babel, (A. Berman et alii), Paris, Trans-Europ-Repress, 1985.

5. BRODA, M. (sous la direction de), La Traduction-Poésie. A Antoine Berman, Strasbourg, Presses Universitaires de Strasbourg, 1999.

6. DASTUR, F., «La pensée comme traduction : autour de Heidegger », in Traduire les philosophes, sous la dir. de Jeacques Moutaux et Olivier Bloch, Paris, Publications de la Sorbonne, 2000, pp 469-482.

7. ESCOUBAS, E., «Philosophie, langue et Bildung : la tâche du traducteur (de Heidegger et Benjamin à Humboldt)», in Traduire les philosophes, sous la dir. de Jeacques Moutaux et Olivier Bloch, Paris, Publications de la Sorbonne, 2000, pp 483-496.

8. FREUD, S., (1887-1902), La Naissance de la psychanalyse, trad. Anne Berman, Paris, PUF, Bibliothèque de psychanalyse, Paris, 1956.

9. FREUD, S., L'interprétation des rêves (1900), trad. I. Meyerson, revue par Denise Berger, Paris, PUF, 1926 et 1967.

10. FREUD, S., Cinq leçons sur la psychanalyse (1904), trad. Yves Le Lay et S. Jankelevitch (trad. revue), Payot, 1980.

11. FREUD, S., (1904-1918), « De la psychotérapie », in La Technique psychanalytique, trad. Anne Berman, Paris, PUF, 1953.

12. FREUD, S., « Perspectives d'avenir de la thérapeutique analytique », in La Technique psychanalytique, trad. Anne Berman, Paris, PUF, 1953.

13. FREUD, S., « Remémoration, répétition, perlaboration », in La Technique psychanalytique, trad. Anne Berman, Paris, PUF, 1953.

14. FREUD, S., Cinq psychanalyses (1905-1925), trad. Marie Bonaparte et, Rudolph Loewenstein, Paris, PUF, Bibliothèque de psychanalyse, 1954.

15. FREUD, S., Psychopathologie de la vie quotidienne, trad. S. Jankelevitch (trad. revue), Paris, Payot, 1981.

16. FREUD, S., Le délire et les rêves dans la Gradiva de W. Jensen (1907), trad. Paule Arbex et RoseMarie Zeitlin, Paris, Gallimard, Folio, 1986.

17. FREUD, S., La vie sexuelle (1907-1931), trad. Denise Berger et Jean Laplanche, Paris, PUF, Bibliothèque de psychanalyse, 1969. 
18. FREUD, S., Un souvenir d'enfance de Léonard de Vinci (1910), trad. J. Altoumian, A. et O. Bourguignon, P. Cotet et A. Ranzy, Paris, Gallimard, Connaissance de 1'Inconscient, 1987.

19. FREUD, S., «L'intérêt de la psychanalyse », in Résultats, idées, problèmes I (1890-1920), trad. sous la direction de Jean Laplanche, Paris, PUF, Bibliothèque de psychanalyse, 1984.

20. FREUD, S., « L'inconscient », in Métapsychologie (1915), trad. J. Laplanche et J.B. Pontalis, Paris, Gallimard, Folio, 1968.

21. FREUD, S., Introduction à la psychanalyse (1915-1916), trad. S. Jankélévitch, Paris, Payot, 1962.

22. FREUD, S., «Remarques sur la théorie et la pratique de l'interprétation des rêves » in Résultats, idées, problèmes II (1921-1938), trad. sous la direction de Jean Laplanche, Paris, PUF, Bibliothèque de psychanalyse, 1985.

23.FREUD, S., Abrége de psychanalyse (1946), trad. Anne Berman, revue et corrigée par Jean Laplanche, Paris, PUF, Bibliothèque de psychanalyse, 1949 et 1985.

24. GRAHAM, J. F., Difference in Translation, Cornell University Press, Ithaque et Londres, 1985.

25. GREEN, A. , « Du comportement à la chair : itinéraire de Merleau-Ponty », in Critique $n^{\circ} 211$, 1964, Paris, pp 1017-1046.

26. GUEST, G., « Une traduction phénoménologique est-elle possible? », in Traduire les philosophes, sous la dir. de Jeacques Moutaux et Olivier Bloch, Paris, Publications de la Sorbonne, 2000, pp 173-199.

27. HASSOUN, J., L'Exil de la langue, Paris, Points Hors Ligne, 1993.

28. LACAN, J., «Fonction et Champ de la parole et du langage », in Ecrits, Paris, éd. Seuil, 1966.

29. LACAN, J., « Maurice Merleau-Ponty », in Autres écrits, Paris, Seuil, 2001.

30. LACAN, J., Les Quatre concepts fondamentaux de la psychanalyse, Paris, Seuil, 1973.

31. MERLEAU-PONTY, M., Phénoménologie de la perception, Paris, Gallimard, 1945,.

32. MERLEAU-PONTY, M., « Le doute de Cézanne », in Sens et non-sens, Paris, Gallimard, 1996.

33. MERLEAU-PONTY, M., Le Visible et l'invisible, Paris, Gallimard, 1960.

34. MERLEAU-PONTY, M., Résumés de cours. Collège de France. 1952-1960, Paris, Gallimard, 1968.

35. MERLEAU-PONTY, M., La Prose du monde, Paris, Gallimard, 1969.

36. MOUNIN, G., Les Problèmes théoriques de la traduction, Paris, Gallimard, 1969.

37. POLITZER, G., Critique des fondements de la psychologie, Paris, PUF, 1974.

38. PONTALIS, J. B., « La Position du problème de l'inconscient chez Merleau-Ponty », in Après Freud, Gallimard, 1993, pp 76-97.

39. PONTALIS, J. B., «Présence, entre les signes, absences », in L'Arc, « Merleau-Ponty », 1971, Paris, pp 56-66. 
40. PONTALIS, J. B., Perdre de vue, Paris, Gallimard, 1988. 